\title{
Shannon sampling and nonlinear dynamics on graphs for representation, regularization and visualization of complex data
}

\author{
M. Pesenson ${ }^{\mathrm{a}}$, I. Pesenson ${ }^{\mathrm{b}}$, B. McCollum ${ }^{\mathrm{a}}$, M. Byalsky ${ }^{\mathrm{c}}$ \\ ${ }^{a}$ California Institute of Technology, m/c 220-6, Pasadena, CA 91125; \\ ${ }^{\mathrm{b}}$ Temple University, Philadelphia; ${ }^{\mathrm{c}}$ The Hebrew University of Jerusalem
}

\begin{abstract}
Data is now produced faster than it can be meaningfully analyzed. Many modern data sets present unprecedented analytical challenges, not merely because of their size but by their inherent complexity and information richness. Large numbers of astronomical objects now have dozens or hundreds of useful parameters describing each one. Traditional color-color plots using a limited number of symbols and some color-coding are clearly inadequate for finding all useful correlations given such large numbers of parameters. To capitalize on the opportunities provided by these data sets one needs to be able to organize, analyze and visualize them in fundamentally new ways. The identification and extraction of useful information in multiparametric, high-dimensional data sets - data mining - is greatly facilitated by finding simpler, that is, lower-dimensional abstract mathematical representations of the data sets that are more amenable to analysis. Dimensionality reduction consists of finding a lower-dimensional representation of high-dimensional data by constructing a set of basis functions that capture patterns intrinsic to a particular state space. Traditional methods of dimension reduction and pattern recognition often fail to work well when performed upon data sets as complex as those that now confront astronomy. We present here our developments of data compression, sampling, nonlinear dimensionality reduction, and clustering, which are important steps in the analysis of large-scale, complex datasets.
\end{abstract}

Keywords: data mining, data compression, graph, sampling, harmonic analysis, unsupervised learning, clustering, synchronization.

\section{INTRODUCTION}

Data representation and retrieval together with detection, characterization and classification of various features of interest are of paramount importance to astronomy. However, high dimensionality complicates these tasks and can easily thwart the entire effort ${ }^{1,2}$. Thus, in order to make practical the extraction of meaningful structures from multiparametric, high-dimensional data sets, a low-dimensional representation of data is required. However, it is important that the representations used cause little or no information loss, which can be a mathematically nontrivial requirement. Classical linear dimension reduction methods like principal component analysis (PCA) and multidimensional scaling (MDS) are not effective in capturing the nonlinear meaningful structures in the data. Unsupervised learning is used to discover distinct subclasses (clusters or groups of patterns), thus providing understanding of the underlying structure of the data. The results of clustering can also be used for classification and to generate hypotheses. Thus unsupervised learning in general and clustering in particular are crucial for statistical data analysis and data mining of large and complex data sets. Traditional clustering methods like $K$-means use a spherical metric to group data points and as a result, they do not work when the clusters are non-convex (see figures in Sec. 3). To overcome some of these limitations, we have been developing Shannon sampling, discrete Fourier transform, splines, tight frames, etc. on manifolds and combinatorial graphs ${ }^{3-11}$, thus extending analysis from Euclidean spaces to curved spaces and laying down theoretical foundations as well as practical algorithms for more adequate representation, effective data retrieval, and analysis of large and complex astronomical data sets. In what follows we describe various data structure and the approaches to sampling, data compression, representation and unsupervised learning that we have developed and are developing in the course of this ongoing research. 


\section{DATA AND GRAPHS}

In the context of multiple problems arising in sampling, computer graphics, data visualization, learning theory and nonlinear data dimension reduction, methods based on graph theory have proved to be very powerful. In mathematics and computer science, graph theory is the study of mathematical structures to model relations between objects ${ }^{13}$. Graph theory has been successfully applied to a wide range of very different disciplines, from biology to social science, computing and physics. Among many applications, graphs and manifolds have been used to address mining databases, Internet search engines, computer graphics, computation reordering, image processing, etc.

\subsection{Graphs, Dimension Reduction and Data Compression}

The graph representation of structured data provides a fruitful model for the relational data mining process. A graph is a collection of nodes and links between them; the nodes represent data points and the weights of the links or edges indicate the strength of relationships. A graph in which each graph edge is replaced by a directed graph edge is called a directed graph, or diagraph. Diagraphs are used for context-sensitive browsing engines and for ranking hyper-linked documents for a given query.

The first important, and very challenging, step is to convert such a data cloud to a weighted graph G. The weight function describes a notion of "similarity" between the data points and as such strongly affects the analysis of the data. The choice of the weight function should take into account a prior knowledge of the data and should entirely be determined by application domain. The most obvious way to assign weights is to use a positive kernel like an exponential function whose exponent depends on the local Euclidean distance between data points and a subjectively chosen parameter called "bandwidth". The recently suggested "diffusion distances" on a manifold or graph ${ }^{14}$ is a powerful approach to the problem. In the latter case a similarity (closeness, weight) of two vertices is estimated by taking into account the geometry of entire graph. This way of assigning weights is in a sense a generalization of how it is done by the well-known PageRank algorithm of the Google search engine. After constructing a weighted graph $\mathrm{G}$ that "represents" a Riemannian manifold M, one can introduce the corresponding combinatorial Laplace operator L, and use its eigenfunctions as a basis. The Laplacian of a graph is the discrete analogue of the Laplace-Beltrami operator on manifolds, and the eigenfunctions of the latter operator have properties desired for embeddings. This is a very active and fruitful approach to the problem of non-linear dimensionality reduction ${ }^{15,14}$. These methods allow one to perform a nonlinear dimension reduction by providing a parameterization of the data set that preserves neighborhoods. The spectral approach revealed that knowledge of the first few eigenfunctions of the corresponding Laplace operators is crucial for multiscale harmonic analysis and effective nonlinear embeddings. The sets of eigenfunctions and eigenvalues of the combinatorial Laplace operator encode important geometric information about the graph that represents a data cloud, thus providing an effective approach for dimension reduction. Thus, to extract information from such data sets requires development of harmonic analysis on graphs and manifolds. By further developing harmonic analysis on graphs and manifolds we have devised innovative algorithms for data compression and nonlinear data dimension reduction ${ }^{3-12}$. These results enable one to overcome PCA's limitations for handling nonlinear data manifolds and also allow one to deal effectively with incomplete data (such as missing observations or partial sky coverage).

Our approach to data compression and dimensionality reduction is based on our discovery of sparse sampling sets for bandlimited functions on manifolds, quantum and combinatorial graphs ${ }^{3-11}$. The compression is not lossless, however, our technique allows a complete reconstruction of all lower frequencies up to certain level that can be chosen in advance. The approach to sampling is based on our innovative methods of reconstruction of bandlimited functions from their sampling sets. Namely, we proved that bandlimited functions on manifolds, quantum and combinatorial graphs, and some fractals can be perfectly reconstructed from specific sampling sets as limits of variational splines. Our new method of reconstruction is based on splines and provides computational advantages over the approach based on diffusion wavelets, since the splines can explicitly be calculated by solving certain linear systems of equations and do not require the knowledge of the eigenfunctions. As we have previously shown, variational splines allow approximate reconstruction (with any degree of accuracy) of the eigenfunctions and eigenvalues of the corresponding Laplace operator. Moreover, it was shown that to reconstruct low eigenvalues and corresponding eigenfunctions only a small portion of the graph is required, thus greatly reducing the computational load for large data sets. This result is a 
generalization of the Shannon sampling theorem. Another advantage of our splines is that unlike the eigenfunctions, they have very strong localization (similar to Euclidean variational splines) in contrast to eigenfunctions. This localization property of our splines allows one to develop a local analysis on graphs. Concepts and methods necessary for such analysis on compact and non-compact manifolds, quantum and combinatorial undirected graphs were first developed by I.Pesenson ${ }^{3-9}$.

\subsection{Shannon sampling on manifolds and graphs}

In this section we show that analysis of lower frequencies on a graph can be performed on a smaller subgraph. By lower frequencies we mean lower eigenvalues of the combinatorial Laplace operator that is introduced below. This main result immediately opens a way to perform data compression and dimension reduction. Note that by ignoring higher frequencies we also smooth data. Moreover, we show that three different and important tasks - denoising, data compression and dimensionality reduction - can be done simultaneously by generalizing the ideas of Shannon's sampling to graphs and manifolds. We consider finite or infinite weighted graphs $G=(V(G), E(G))$, where $V(G)$ is its set of vertices and $E(G)$ is its set of edges. The space $L_{2}(G)$ is the Hilbert space of all complex-valued functions on $V(G)$ with the natural inner product and the corresponding norm $\|\mathrm{f}\|$. If $\mathrm{w}(\mathrm{v}, \mathrm{u})$ is a weight associated with vertices $\mathrm{v}$ and $\mathrm{u}$, then the corresponding Laplace operator is introduced by the formula

$$
\operatorname{Lf}(\mathrm{v})=\sum_{\mathrm{u}, \mathrm{v} \sim \mathrm{u}}[f(\mathrm{v})-\mathrm{f}(\mathrm{u})] \mathrm{w}(\mathrm{v}, \mathrm{u})
$$

The Laplace operator $\mathrm{L}$ is self-adjoint and positive definite in the space $\mathrm{L}_{2}(\mathrm{G})$. For a finite connected graph the spectrum of $\mathrm{L}$ is of the form $0=\lambda_{0}<\ldots<\lambda_{\mathrm{n}-1}$ where $\mathrm{n}$ is a number of vertices. Let $\Phi=\left\{\varphi_{0}, \ldots, \varphi_{\mathrm{n}-1}\right\}$ be a corresponding system of orthonormal eigenfunctions. Given a positive $\omega>0$ the subspace $E_{\omega}$ of $\omega$-bandlimited functions is the span of such functions in $\Phi$ whose corresponding eigenvalues are not greater than $\omega$. Furthermore, based on the spectral theorem for self-adjoint operators we introduced a new concept of bandlimitedness for the case of infinite graphs ${ }^{3,4}$. This enables us to extend Shannon sampling theory to infinite graphs.

To illustrate our sampling results let us recall the classical Shannon-Nyquist sampling theorem. It states that for all bandlimited functions of a fixed bandwidth defined on Euclidean space one can find "not very dense" sampling sets, which can be used to represent all relevant bandlimited functions. In some sense it allows one to reduce the set of all points of Euclidean space to a countable set of points. To demonstrate our approach let us consider a simple example. Suppose that a data set is composed of $10^{6}$ points. Let us assume for simplicity that we identify our data set with the path graph $\mathrm{Z}$ of $10^{6}$ vertices. We measure frequency on this graph in terms of the eigenvalues of the corresponding Laplace operator on $\mathrm{Z}$. It has $10^{6}$ eigenvalues (frequencies) which all belong to the interval $[0,4]$ and are given by the formula 2 $2 \cos \left[\pi \mathrm{k} /\left(10^{6}-1\right)\right], \mathrm{k}=0,1, \ldots, 10^{6}-1$. Our results show that if one deletes every second point from the $10^{6}$ vertices, then the resulting set is still sufficient for reconstruction of $\sim 10^{5}$ first eigenfunctions. If one deletes about $2 / 3$ of all points then the resulting set is a sampling set for about the first $6 \times 10^{4}$ first eigenfunctions. By extending our reasoning it is possible to show that about 10 percent of "uniformly distributed" points of $\mathrm{Z}$ form a sampling set for the about 500 first eigenfunctions. It is important to keep in mind that in reality the amount of information one wishes to discard depends on a particular user's goals. We would like to emphasize that our approach also provides a way to estimate the error of the reconstruction procedure.

The idea of sampling on manifolds and graphs can be used for data compression. For the sake of simplicity we consider a combinatorial (finite or infinite) graph. Let $F=\left(f_{1}, \ldots, f_{N}\right)$ be an embedding of a graph $G$ into a high-dimensional Euclidean space $\mathrm{R}^{\mathrm{N}}$. Let $\mathrm{L}$ be the Laplace operator on $\mathrm{G}$ and $\mathrm{E}_{\omega}$ be a subspace of bandlimited functions associated with L. By using our concept of bandlimited function on a graph we project each function $f_{j}$ onto $E_{\omega}$ and obtain a new filtered embedding $F^{\omega}=\left(f_{1}{ }_{1}^{\omega} \ldots, f_{N}{ }^{\omega}\right)$ of $G$ into $R^{N}$ with reduced noise. Since $F^{\omega}$ is bandlimited it is completely determined by its values on a relatively small subset of vertices $\mathrm{U}$, which is a sampling set for $\mathrm{F}^{\omega}$. In other words, one can completely forget about the values of $F^{\omega}$ on the rest of the graph $V(G) \backslash U$ and to utilize only the values of $F^{\omega}$ on $U$, since $F^{\omega}$ has a unique $\omega$-bandlimited extension to entire graph $\mathrm{G}$. In the case of a finite graph $\mathrm{G}$ the size of such a sampling set $\mathrm{U}$ is comparable with the number of eigenvalues of $\mathrm{L}$ that belong to $[0, \omega]$. Let us stress that we can completely reconstruct all the lower frequencies $\leq \omega$ by using a restriction of the Laplacian to a relatively small sampling set $\mathrm{U}$. We also derived explicit estimates of the accuracy of this reconstruction. 
The methods described in this section offer savings in data storage space and database-transmission bandwidth. Moreover, they greatly increase computational efficiency of machine learning algorithms and improve statistical inference, thus facilitating automated feature selection, data segmentation, classification and effective scientific visualization that are discussed in the next section.

\section{UNSUPERVISED LEARNING AND DATA CLUSTERING}

Unsupervised learning in general and clustering (automatic identification of groups of similar objects) and classification (assigning labels to instances) in particular are crucial for multiple problems arising in pattern recognition, data mining, inference and prediction from data, computer graphics and data visualization. Traditional methods, like the staple of clustering techniques the $K$-means algorithm ${ }^{16}$, use a spherical metric to group data points. As a result, they do not work when the clusters are non-convex (see Figures below). There are other approaches to clustering based on analogies with various physical phenomena. For example, Horn and Gottlieb ${ }^{17}$ developed an approach that is based on the Schrödinger operator, where they constructed an approximate eigenfunction, and computed its corresponding potential. The clusters of data points were then defined by the minima of the potential. Another approach ${ }^{18}$ is based on associating points with Potts spins and formulating an appropriate model of statistical mechanics. We developed an alternative that is also based on physical intuition, this one being derived from synchronization of nonlinear oscillations ${ }^{19,20}$. Approximating a multidimensional image or a data set by a graph (Sec. 2) and associating a nonlinear dynamical system with each node enables us to cluster data effectively. The frequencies and intersections with the axis $\mathrm{t}=0$ of the asymptotic phases (Fig. 1 , right) of the dynamical system may be used for mapping the data, thus providing a new mechanism for nonlinear data dimension reduction. Figures 1-5. illustrate applications of the dynamical clustering to simulated 2D and 3D non-convex data.
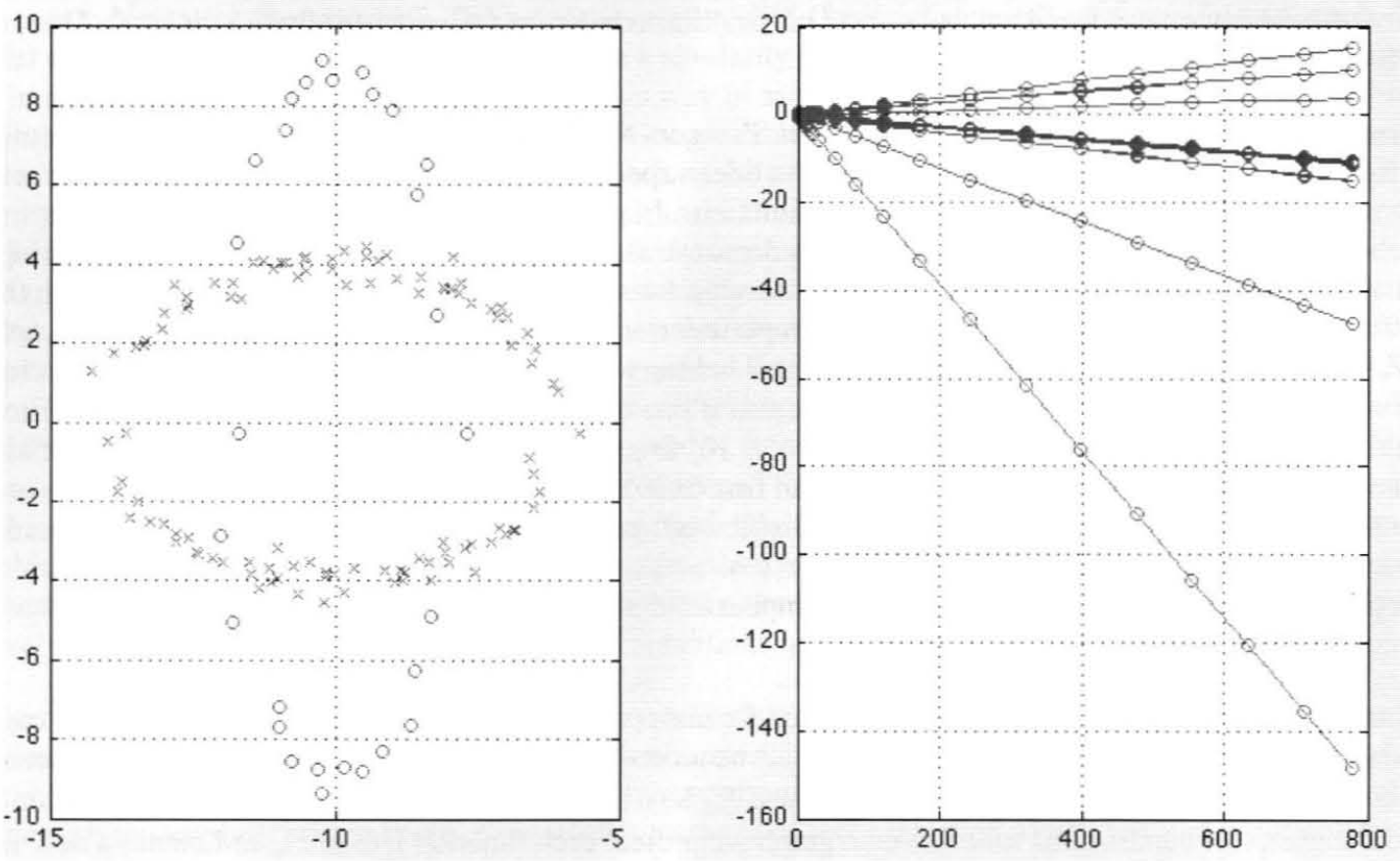

Figure 1. Dynamical clustering in 2D. Left: blue and red (the color version is available on line) data points sampled from two intersecting ellipses with added Gaussian noise. Right: phases of the dynamical system associated with the graph to which the data set was converted; the blue points all have the same frequency $\omega$ and applying a standard (e.g., $K$-means) clustering in this new representation (in p- $\omega$ plane) will easily recover the original clusters. 

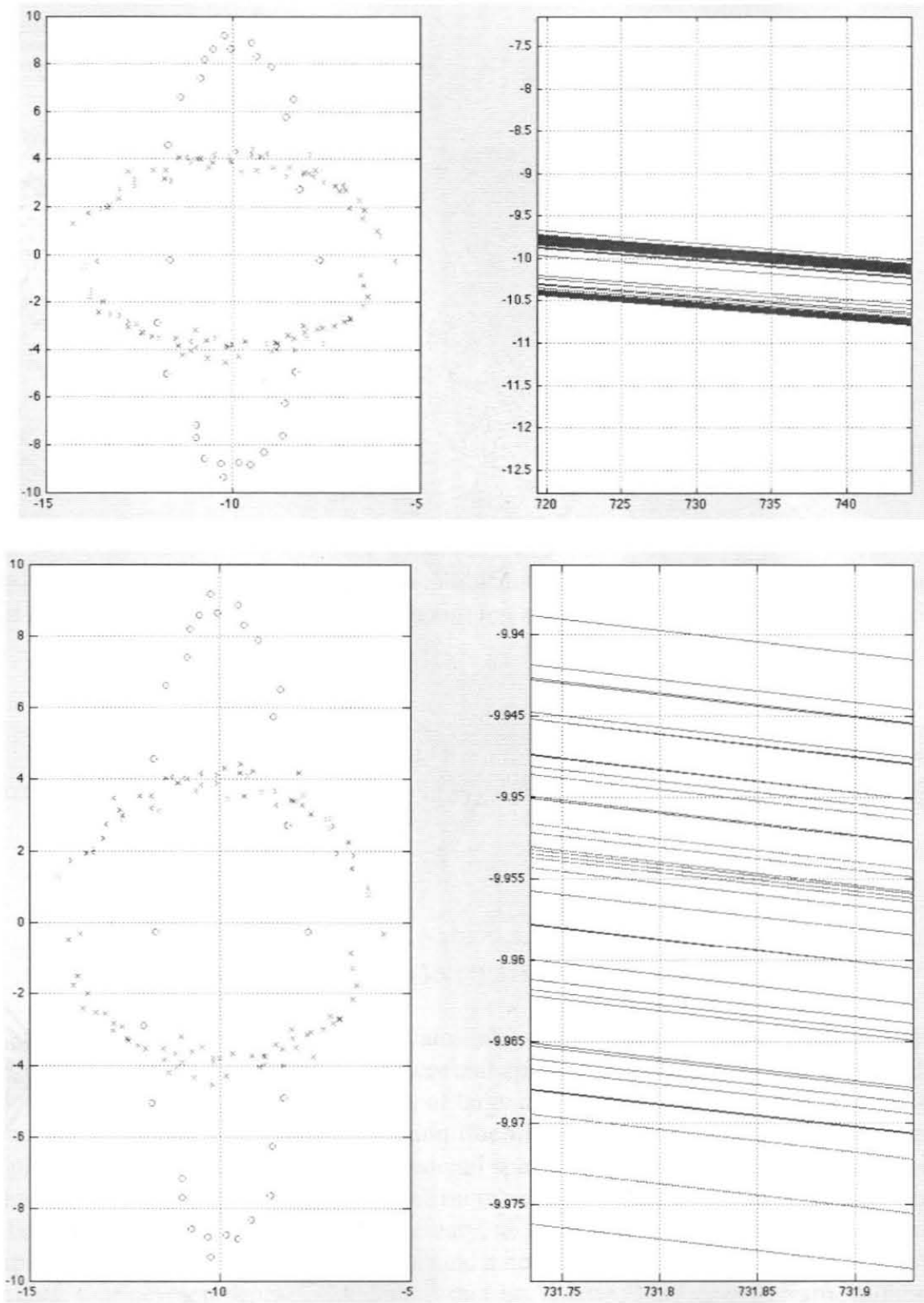

Figure 2. Same as Fig. 1., with the phases progressively zoomed in. The right hand side shows that even though the clusters are quite close to each other, only two red points got mixed with the blue points (the color version is available on line). 

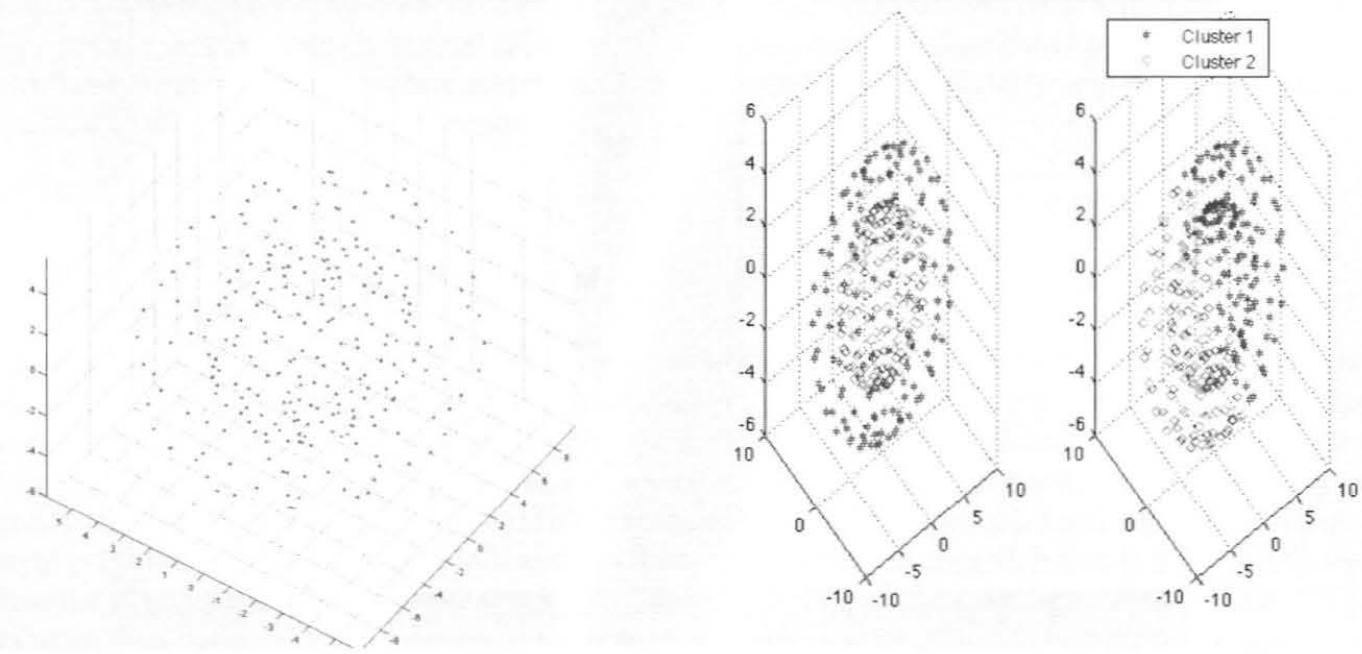

Figure 3. Clustering in 3D. Left: a simulated data set. Middle: dynamical clustering reveals two concentric ellipsoids. Right: the result of K-means clustering - the clusters got mixed (the color version is available on line).
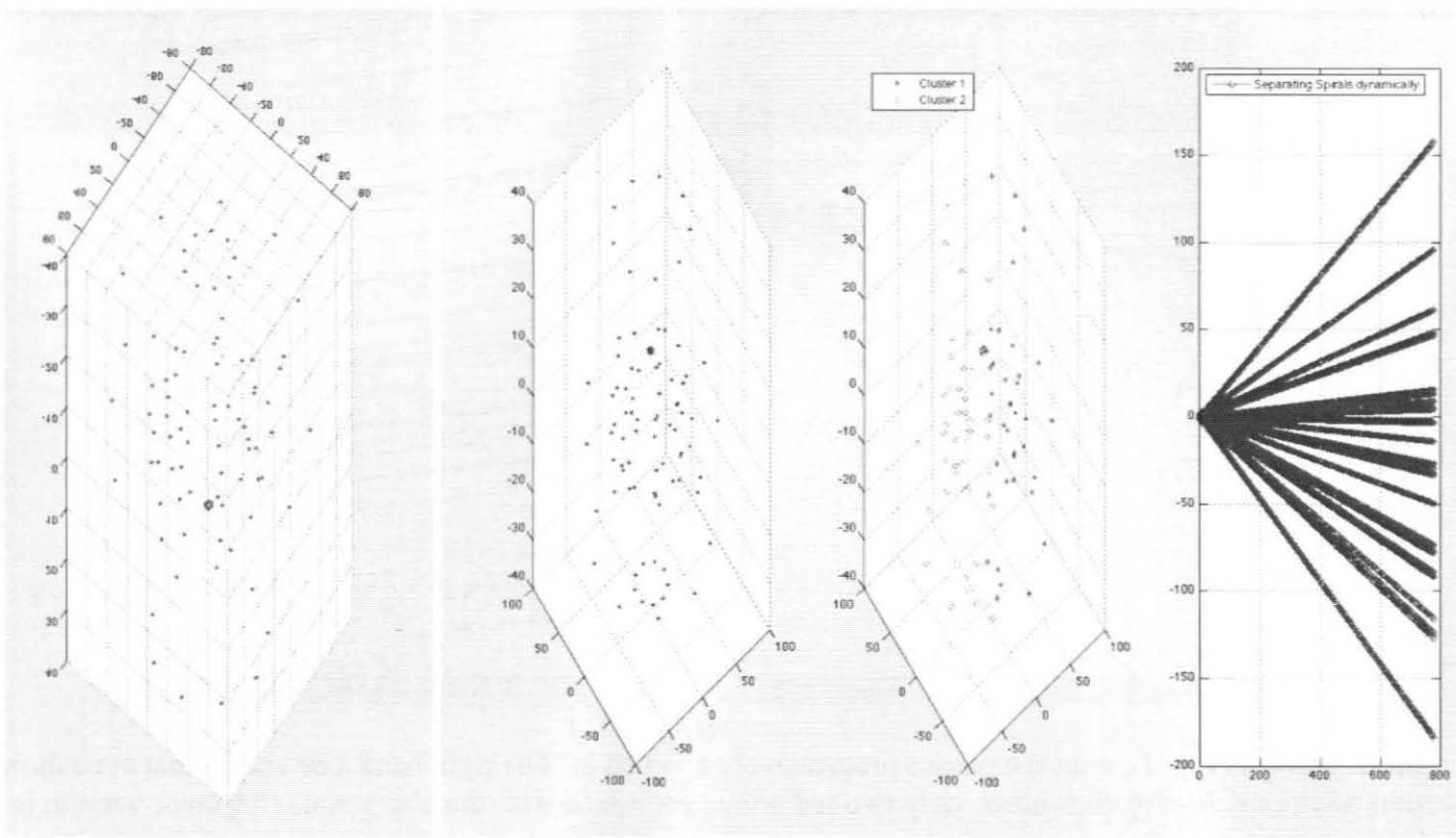

Figure 4. Clustering in 3D. Left: a simulated data set. Right (first plot): dynamical clustering reveals two ellipsoidal embeded toruses (see the next figure). Right (second plot): the result of K-means clustering - the clusters got mixed. Right (third plot): phases of the nonlinear dynamical system. Again, applying a standard (e.g., $K$-means) clustering in this new representation (in $\mathrm{p}-\omega$ plane) will easily recover the original clusters (the color version is available on line). 


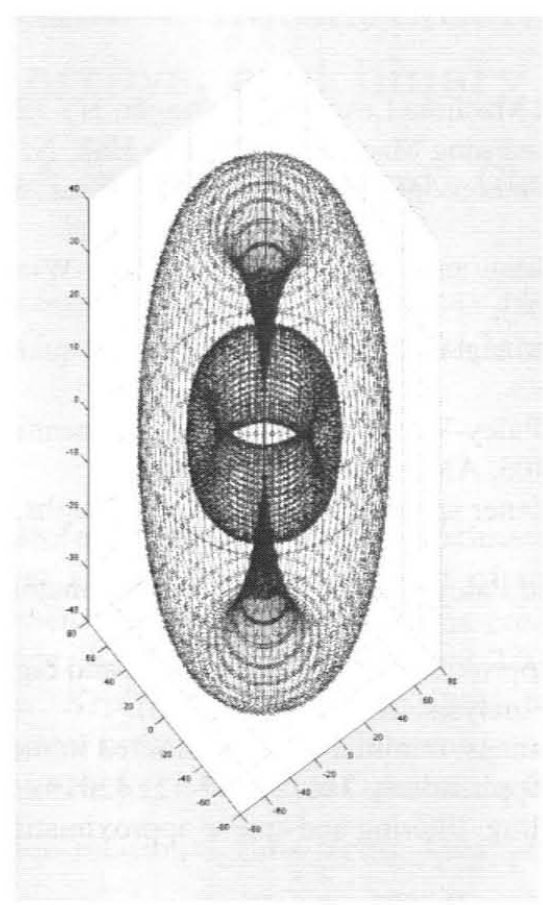

Figure 5. The manifolds from which the data points in Fig.4(left) were sampled (the color version is available on line).

\section{CONCLUSION}

The complexity of many new astronomical data sets creates new challenges to data mining and analysis that cannot always be met by simply scaling up raw computing power and applying familiar analysis methods. In this paper we presented new approaches that enable practical analysis of large and complex data sets having hundreds of parameters for each object, by mapping the data into lower-dimension Euclidean spaces where analysis becomes much more feasible. For example, when performed in lower dimensional spaces object classification based on simultaneously evaluating large numbers of parameters can then be done more reliably than in high dimensional spaces. This sort of data reduction can be significantly beneficial, even necessary, as a pre-processing step for many existing astronomical packages, for example SExtractor ${ }^{21}$. We have also presented a new approach to unsupervised learning. and visualization of many-parameter data. Our developments of Shannon sampling, discrete Fourier transform, splines, tight frames, wavelets, etc. on manifolds and combinatorial graphs ${ }^{3-11}$ extend analysis from Euclidean spaces to curved spaces and lay down theoretical foundations as well as practical algorithms for more adequate representation, effective data retrieval, and practical analysis of complex astronomical data sets. In addition to astrophysical applications, the described methods find applications in common multicomputer networks used for grid computing, communication networks (WWW, Internet, power grid, etc.), computer graphics, etc.

\section{Acknowledgement}

This work was carried out with funding from the National Geospatial-Intelligence Agency University Research Initiative (NURI), grant HM1582-08-1-0019, and support from NASA to the California Institute of Technology and the Jet Propulsion Laboratory. 


\section{REFERENCES}

[1] Bishop C., [Pattern Recognition and Machine Learning], Springer, NY (2007).

[2] Haykin, S., [Neural Networks and Learning Machines], Prentice Hall, NJ (2009).

[3] Pesenson, I., "A sampling theorem on homogeneous manifolds," Trans. of American Math. Soc, 352(9), 4257-4270 (2000).

[4] Pesenson, I., "Poincare-type inequalities and reconstruction of Paley-Wiener functions on manifolds," J. of Geometric Analysis, 4(1), 101-121 (2004).

[5] Pesenson, I., "Polynomial splines and eigenvalue approximations on quantum graphs," J. Approx. Theory, v.135, no. 2, 203-220 (2005).

[6] Pesenson, I., "Frames for spaces of Paley-Wiener Functions on Riemannian manifolds," Integral Geometry and Tomography, Contemp. Mathematics, 405, AMSoc., 135-148 (2006).

[7] Pesenson, I., "Sampling in Paley-Wiener spaces on combinatorial graphs," Trans. Amer. Math. Soc. 360, no. 10, 5603-5627 (2008).

[8] Pesenson, I., "Variational splines and Paley-Wiener spaces on combinatorial graphs," Constructive Approximation, 29, no. 1, 1-20 (2009).

[9] Pesenson, I., "Removable sets and approximations of eigenvalues and eigenfunctions on combinatorial graphs," Applied and Computational Harmonic Analysis, 29, 123-133 (2010).

[10] Pesenson, I., Pesenson, M., "Eigenmaps, minimal and bandlimited immersions of graphs into Euclidean spaces," Journal of Mathematical Analysis and Applications, 366, 1, 137-152 (2010).

[11] Pesenson, I., Pesenson, M., "Sampling, filtering and sparse approximations on graphs," Journal of Fourier Analysis and Applications, (accepted) (2010).

[12] Pesenson, M., Pesenson, I., McCollum, B., "The data Big Bang and the expanding digital Universe: High-

Dimensional, Complex and Massive Data Sets in an Inflationary Epoch," Advances in Astronomy, Special issue on Robotic Astronomy, v. 2010, Article ID 350891 (2010).

[13] Borgelt, C., Steinbrecher, A., Kruse, R., [Graphical Models. Representations for Learning, Reasoning and Data Mining],Wiley, UK (2009).

[14] Coifman, R., Lafon, S., Lee, A., et al., "Geometric diffusions as a tool for harmonic analysis and structure definition of data," Proceedings of the National Academy of Sciences of the USA, 102, 7426-7431 (2005).

[15] Belkin, M., Niyogi, P., "Towards a theoretical foundation for Laplacian-based manifold methods, Learning Theory," Lecture Notes in Computational Science, 3559, 486-500, Springer, Berlin (2005).

[16] Duda, R., Hart, P., Stork, D., [Pattern Classification], Wiley, NY (2001).

[17] Horn, D., Gottlieb, A. "Algorithm for Data Clustering in Pattern Recognition Problems Based on Quantum Mechanics.” Phys. Rev. Lett., 88, 018792 1-4 (2002).

[18] Blatt M., Wiseman, S., Domany, E., "Superparamagnetic Clustering of Data", Phys. Rev. Lett., 76, 18, 3251-3254

(1996).

[19] Kuramoto, Y., [Chemical Oscillations, Waves and Turbulence], Springer, Berlin (1984).

[20] Pikovsky, A., Rosenblum, M. \& Kurths, J., [Synchronization], Cambridge UP (2001).

[21] Bertin, E., Arnouts, A., "SExtractor: Software for Source Separation," A\&A, 117, 939 (1996). 